Pacific Journal of Mathematics

BLOCK DIAGONALLY DOMINANT MATRICES AND
GENERALIZATIONS OF THE GRSCHGORIN CIRCLE
THEOREM

David G. Feingold AND Richard STEVEn VARG 


\title{
BLOCK DIAGONALLY DOMINANT MATRICES AND GENERALIZATIONS OF THE GERSCHGORIN CIRCLE THEOREM
}

\author{
David G. Feingold and Richard S. VARga
}

1. Introduction. The main purpose of this paper is to give generalizations of the well known theorem of Gerschgorin on inclusion or exclusion regions for the eigenvalues of an arbitrary square matrix $A$. Basically, such exclusion regions arise naturally from results which establish the nonsingularity of $A$. For example, if $A=D+C$ where $D$ is a nonsingular diagonal matrix, then Householder [7] shows that $\left\|D^{-1} C\right\|<1$ in some matrix norm is sufficient to conclude that $A$ is nonsingular. Hence, the set of all complex numbers $z$ for which

$$
\left\|(z I-D)^{-1} C\right\|<1
$$

evidently contains no eigenvalues of $A$. In a like manner, Fiedler [4] obtains exclusion regions for the eigenvalues of $A$ by establishing the nonsingularity of $A$ through comparisons with $M$-matrices. ${ }^{1}$ Our approach, though not fundamentally different, establishes the nonsingularity of the matrix $A$ by the generalization of the simple concept of a diagonally dominant matrix. But one of our major results $(\S 3)$ is that these new exclusion regions can give significant improvements over the usual Gerschgorin circles in providing bounds for the eigenvalues of $A$.

2. Block diagonally dominant matrices. Let $A$ be any $n \times n$ matrix with complex entries, which is partitioned in the following manner:

$$
A=\left[\begin{array}{cccc}
A_{1,1} & A_{1,2} & \cdots & A_{1, N} \\
A_{2,1} & A_{2,2} & \cdots & A_{2, N} \\
\vdots & & & \vdots \\
A_{N, 1} & A_{N, 2} & \cdots & A_{N, N}
\end{array}\right],
$$

where the diagonal submatrices $A_{i, i}$ are square of order $n_{i}, 1 \leqq i \leqq N$. For reasons to appear in $\S 3$, the particular choice $N=1$ of

$$
A=\left[A_{1,1}\right]
$$

will be useful. Viewing the square matrix $A_{i, i}$ as a linear transformation of the $n_{i}$-dimensional vector subspace $\Omega_{i}$ into itself, we associate with this subspace the vector norm $\|\boldsymbol{x}\|_{\Omega_{i}}$, i.e., if $\boldsymbol{x}$ and $\boldsymbol{y}$ are elements of

Received April 11, 1962.

${ }^{1}$ For the definition of an $M$-matrix, see $\& 4$ or [8]. 
$\Omega_{i}$, then

$$
\left\{\begin{array}{l}
\|\boldsymbol{x}\|_{\Omega_{i}}>0 \text { unless } \boldsymbol{x}=\boldsymbol{O} ; \\
\|\alpha \boldsymbol{x}\|_{\Omega_{i}}=|\alpha|\|\boldsymbol{x}\|_{\Omega_{i}} \text { for any scalar } \alpha ; \\
\|\boldsymbol{x}+\boldsymbol{y}\|_{\Omega_{i}} \leqq\|\boldsymbol{x}\|_{\Omega_{i}}+\|\boldsymbol{y}\|_{\Omega_{i}}, \quad 1 \leqq i \leqq N .
\end{array}\right.
$$

The point here is that we can associate different vector norms with different subspaces $\Omega_{i}$. Now, similarly considering the rectangular matrix $A_{i, j}$ for any $1 \leqq i, j \leqq N$ as a linear transformation from $\Omega_{j}$ to $\Omega_{i}$, the norm $\left\|A_{i, j}\right\|$ is defined as usual by

$$
\left\|A_{i, j}\right\| \equiv \sup _{\boldsymbol{x} \in \Omega_{j}, \boldsymbol{x} \neq \boldsymbol{o}} \frac{\left\|A_{i, j} \boldsymbol{x}\right\|_{\Omega_{i}}}{\|\boldsymbol{x}\|_{\Omega_{j}}}
$$

Note that if the partitioning in (2.1) is such that all the matrices $A_{i, j}$ are $1 \times 1$ matrices and $\|\boldsymbol{x}\|_{\Omega_{i}} \equiv|x|$, then the norms $\left\|A_{i, j}\right\|$ are just the moduli of the single entries of these matrices. As no confusion arises, we shall drop the subscripts on the different vector norms. ${ }^{2}$

Definition 1. Let the $n \times n$ matrix $A$ be partitioned as in (2.1). If the diagonal submatrices $A_{j, j}$ are nonsingular, and if

$$
\left(\left\|A_{j, j}^{-1}\right\|\right)^{-1} \geqq \sum_{\substack{k=1 \\ k \neq j}}^{N}\left\|A_{j, k}\right\| \quad \text { for all } 1 \leqq j \leqq N,
$$

then $A$ is block diagonally dominant, relative to the partitioning (2.1). If strict inequality in (2.4) is valid for all $1 \leqq j \leqq N$, then $A$ is block strictly diagonally dominant, relative to the partitioning of (2.1).

It is useful to point out that the quantity appearing on the lefthand side of (2.4) can also be characterized form (2.3) by

$$
\left(\left\|A_{j, j}^{-1}\right\|\right)^{-1}=\inf _{\boldsymbol{x} \in \Omega_{j}, \boldsymbol{x} \neq \boldsymbol{o}}\left(\frac{\left\|A_{j, j} \boldsymbol{x}\right\|}{\|\boldsymbol{x}\|}\right),
$$

whenever $A_{j, j}$ is nonsingular. With (2.5), we can then define $\left(\left\|A_{j, j}^{-1}\right\|\right)^{-1}$ by continuity to be zero whenever $A_{j, j}$ is singular.

In the special case that all the matrices $A_{i, j}$ are $1 \times 1$ matrices and $\|x\|=|x|$, then (2.4) can be written as

$$
\left|A_{j, j}\right| \geqq \sum_{\substack{k=1 \\ k \neq j}}^{N}\left|A_{j, k}\right| \text { for all } 1 \leqq j \leqq N,
$$

which is the usual definition of diagonal dominance.

As an example of a matrix which is block strictly diagonally dominant, consider the case $n=4, N=2$ of

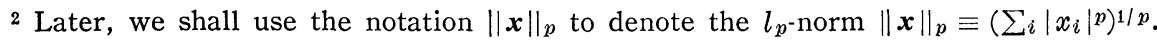




$$
A=\left[\begin{array}{cc|cc}
0 & 1 & 0 & 0 \\
2 / 3 & 0 & 1 / 3 & 0 \\
\hline 0 & 1 / 3 & 0 & 2 / 3 \\
0 & 0 & 1 & 0
\end{array}\right],
$$

where we choose the vector norms $\|x\|_{\infty} \equiv \max _{j}\left|x_{j}\right|$. In this case,

$$
\left(\left\|A_{1,1}^{-1}\right\|\right)^{-1}=\left(\left\|A_{2,2}^{-1}\right\|\right)^{-1}=\frac{2}{3} \text {, and }\left\|A_{1,2}\right\|=\left\|A_{2,1}\right\|=\frac{1}{3} \text {. }
$$

Obviously, $A$ is not diagonally dominant in the sense of $\left(2.4^{\prime}\right)$.

Definition 2. The $n \times n$ partitioned matrix $A$ of (2.1) is block irreducible if the $N \times N$ matrix $B=\left(b_{i, j} \equiv\left\|A_{i, j}\right\|\right), 1 \leqq i, j \leqq N$, is irreducible, i.e., the directed graph of $B$ is strongly connected. ${ }^{3}$

THEOREM 1. If the partitioned matrix $A$ of (2.1) is block strictly diagonally dominant, or if $A$ is block irreducible and block diagonally dominant with inequality holding in (2.4) for at least one $j$, then $A$ is nonsingular.

Proof. The extension to the case where $A$ is block irreducible and block diagonally dominant with strict inequality for at least one $j$ is easy, so we consider for simplicity only the case when $A$ is block strictly diagonally dominant. Suppose that $A$ is singular, i.e., there exists a nonzero vector $\boldsymbol{W}$ with

$$
A\left[\begin{array}{c}
W_{1} \\
\vdots \\
W_{N}
\end{array}\right]=\boldsymbol{O}
$$

here, we have partitioned $\boldsymbol{W}$ conformally with respect to the partitioning of (2.1). But this is equivalent to

$$
\sum_{\substack{j=1 \\ j \neq i}}^{N} A_{i, j} W_{j}=-A_{i, i} W_{i}, \quad 1 \leqq i \leqq i N
$$

Since $\boldsymbol{W}$ is a nonzero vector, normalize $\boldsymbol{W}$ so that $\left\|W_{j}\right\| \leqq 1$ for all $1 \leqq$ $j \leqq N$, and assume that equality is valid for some $r$, i.e., $\left\|W_{r}\right\|=1$ where $1 \leqq r \leqq N$. Thus, from (2.3)

${ }^{3}$ Equivalently, there exists no $N \times N$ permutation matrix $P$ such that $P B P^{T}=\left[\begin{array}{l}O D \\ O E\end{array}\right]$, where $C$ and $E$ are square nonvoid submatrices. For strongly connected directed graphs, see for example [6]. 


$$
\left\|A_{r, r} W_{r}\right\|=\left\|\sum_{\substack{j=1 \\ j \neq r}}^{N} A_{r, j} W_{j}\right\| \leqq \sum_{\substack{j=1 \\ j \neq r}}^{N}\left\|A_{r, j}\right\| \cdot\left\|W_{j}\right\| \leqq \sum_{\substack{j=1 \\ j \neq r}}^{N}\left\|A_{r, j}\right\|
$$

But as $A_{r, r}$ is nonsingular by hypothesis, then putting $A_{r, r} W_{r}=Z_{r}$,

$$
\left\|A_{r, r} W_{r}\right\|=\frac{\left\|A_{r, r} W_{r}\right\|}{\left\|W_{r}\right\|}=\frac{\left\|Z_{r}\right\|}{\left\|A_{r, r}^{-1} Z_{r}\right\|} \geqq\left(\left\|A_{r, r}^{-1}\right\|\right)^{-1},
$$

using (2.3). This combined with $\left(2.8^{\prime}\right)$ gives a contradiction to the assumption (2.4) that $A$ is block strictly diagonally dominant, which completes the proof for the block strictly diagonally dominant case.

Actually, we can regard Theorem 1 as the block analogue of the well known Hadamard theorem on determinants, since Theorem 1 reduces to this result in the case that all the matrices $A_{i, j}$ of (2.1) are $1 \times 1$ matrices and $\|x\| \equiv|x|$. It should be pointed out that the result of Theorem 1 itself is a special case of a more general result by Ostrowski [10, Theorem 3, p. 185], and Fiedler [4].

As stated in the introduction, the above theorem leads naturally to a block analogue of the Gerschgorin Circle Theorem. If $I$ is the $n \times n$ identity matrix which is partitioned as in (2.1), and $I_{j}$ is the $n_{j} \times n_{j}$ identity matrix, suppose that

$$
\left(\left\|\left(A_{j, j}-\lambda I_{j}\right)^{-1}\right\|\right)^{-1}>\sum_{\substack{k=1 \\ k \neq j}}^{N}\left\|A_{j, k}\right\| \text { for all } 1 \leqq j \leqq N .
$$

Thus, we have from Theorem 1 that $A-\lambda I$ is nonsingular. Hence, if $\lambda$ is an eigenvalue of $A$, then $A-\lambda I$ cannot be block strictly diagonally dominant, which gives us

THEOREM 2. For the partitioned matrix $A$ of (2.1), each eigenvalue $\lambda$ of $A$ satisfies

$$
\left(\left\|\left(A_{j, j}-\lambda I_{j}\right)^{-1}\right\|\right)^{-1} \leqq \sum_{\substack{k=1 \\ k \neq j}}^{N}\left\|A_{j, k}\right\|
$$

for at least one $j, 1 \leqq j \leqq N$.

We again remark that if the partitioning of (2.1) is such that all the diagonal submatrices are $1 \times 1$ matrices and $\|x\| \equiv|x|$, then Theorem 2 reduces to the well known Gerschgorin Circle Theorem.

3. Inclusion regions for eigenvalues. In Theorem 2 , we saw that each eigenvalue $\lambda$ of an arbitrary $n \times n$ complex matrix $A$ necessarily satisfied (2.10) for at least one $j, 1 \leqq j \leqq N$.

Definition 3. For the partitioned $n \times n$ matrix $A$ of (2.1), let the 
Gerschgorin set $G_{j}$ be the set of all complex numbers $z$ such that

$$
\left(\left\|\left(A_{j, j}-z I\right)^{-1}\right\|\right)^{-1} \leqq \sum_{\substack{k=1 \\ k \neq j}}^{N}\left\|A_{j, k}\right\|, \quad 1 \leqq j \leqq N .
$$

Thus, from (2.5), we conclude that the Gerschgorin set $G_{j}$ always contains the eigenvalues of $A_{j, j}$, independent to the magnitude of the right side of (3.1) and independent of the vector norms used. Next, it is clear that each Gerschgorin set $G_{j}$ is closed and bounded. Hence, so is their union

$$
G=\bigcup_{j=1}^{N} G_{j}
$$

Thus, we can speak of the boundary of $G$, as well as the boundary of each $G_{j}$. By Theorem 2, all the eigenvalues of $A$ lie in $G$. Can any eigenvalue $\lambda$ of $A$ lie on the boundary of $G$ ? This can be answered trivially for the particular partitioning of $\left(2.1^{\prime}\right)$. In this case, the righthand side of (3.1) is vacuously zero, and from (3.1), we see that the set $G$ is a finite point set consisting only of the eigenvalues of $A$. In this case, Theorem 2 gives exact information about the eigenvalues of $A$.

It is interesting that Theorem 2 can be strengthened by the assumption that $A$ is block irreducible, which is the analogue of a well known result of Taussky [11].

THEOREM 3. Let the partitioned matrix A of (2.1) be block irreducible, and let $\lambda$ be an eigenvalue of $A$. If $\lambda$ is a boundary point of $G$, then it is a boundary point of each set $G_{j}, 1 \leqq j \leqq N$.

Proof. Since $\lambda$ is an eigenvalue of $A$, then $\sum_{j=1}^{N} A_{i, j} W_{j}=\lambda W_{i}$, and if $\left\|W_{j}\right\| \leqq\left\|W_{r}\right\|=1$, then as before

$$
\left(\left\|\left(A_{r, r}-\lambda I_{r}\right)^{-1}\right\|\right)^{-1} \leqq \sum_{\substack{j=1 \\ j \neq r}}^{N}\left\|A_{r, j}\right\|\left\|W_{j}\right\| \leqq \sum_{\substack{j=1 \\ j \neq r}}^{N}\left\|A_{r, j}\right\| .
$$

But as $\lambda$ is a boundary point of $G$, equality must hold throughout (3.3), showing that $\lambda$ is a boundary point of $G_{r}$. Moreover, if $\left\|A_{r, j}\right\| \neq 0$, then $\left\|W_{j}\right\|=1$, and we can repeat the argument with $r$ replaced by $j$. In this way, we conclude that $\lambda$ is a boundary point of $G_{j}$. From the irreducibility of $A$, the argument can be extended to every index $j$, $1 \leqq j \leqq N$, which completes the proof. A similar argument can be applied to complete the proof of Theorem 1.

Another familiar result of Gerschgorin can also be generalized. The proof, depending on a continuity argument, follows that given in [13, p. 287].

THEOREM 4. If the union $H=\bigcup_{j=1}^{m} G_{p_{j}}, 1 \leqq p_{j} \leqq N$, of $m$ Gerschgorin 
sets is disjoint from the remaining $N-m$ Gerschgorin sets for the partitioned matrix $A$ of (2.1), then $H$ contains precisely $\sum_{j=1}^{m} n_{p_{j}}$ eigenvalues of $A$.

The previous example of the matrix of $\left(2.1^{\prime}\right)$ indicated that sharper inclusion regions for the eigenvalues of a matrix $A$ may be obtained from the generalized form of Gerschgorin's Theorem 2. To give another illustration, consider the partitioned matrix

$$
A=\left[\begin{array}{rr|rr}
4 & -2 & -1 & 0 \\
-2 & 4 & 0 & -1 \\
\hline-1 & 0 & 4 & -2 \\
0 & -1 & -2 & 4
\end{array}\right]=\left[\begin{array}{l|l}
A_{1,1} & A_{1,2} \\
\hline A_{2,1} & A_{2,2}
\end{array}\right]
$$

Employing now the vector norm $\|\boldsymbol{x}\|_{2} \equiv\left(\sum_{i}\left|x_{i}\right|^{2}\right)^{1 / 2}$, it is apparent that $\left\|A_{1,2}\right\|=\left\|A_{2,1}\right\|=1$. On the other hand, direct computation shows that

$$
\left(\left\|\left(A_{i, i}-z I_{i}\right)^{-1}\right\|^{-1}=\min \{|6-z|,|2-z|\}, \quad i=1,2 .\right.
$$

By definition, the set $G_{1}$ then consists of the points $z$ for which

$$
|6-z| \leqq 1, \quad|2-z| \leqq 1,
$$

so that $G_{1}$ is itself the union of two disjoint circles. The same is true for $G_{2}$, since $G_{2}=G_{1}$, as shown in the figure below. The usual Gerschgorin circles

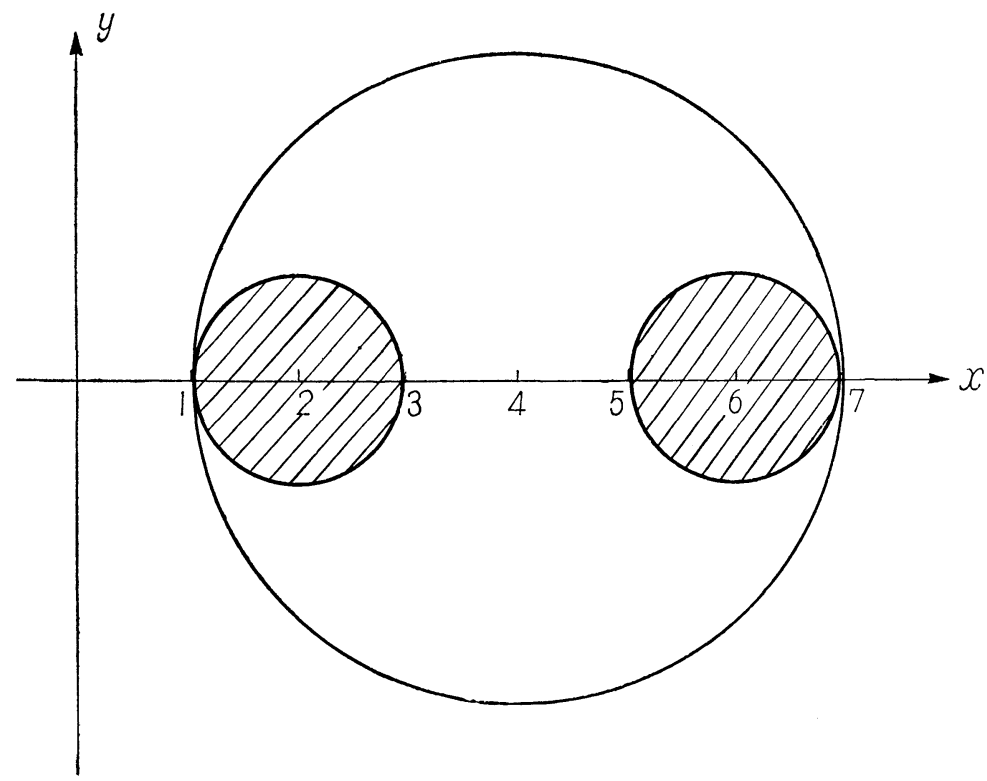


for the matrix $A$ of (3.4) are all given by the single circle $|4-\lambda| \leqq 3$, which is a circle of radius 3 , with center at $z=4$, as shown above. From this figure, we conclude that the block Gerschgorin result can give significant improvements over the usual Gerschgorin circles in providing bounds for eigenvalues. For the matrix $A$ of (3.4), its eigenvalues are

$$
\lambda_{1}=1, \lambda_{2}=3, \lambda_{3}=5, \lambda_{4}=7 .
$$

Note, again from the figure above, that Theorem 3 applies in this case.

At this point, we remark that the previous example was such that each Gerschgorin set $G_{j}$ consisted of the union of circles. This is a special case of

Theorem 5. Let the partitioned matrix $A$ of (2.1) be such that its diagonal submatrices $A_{j, j}$ are all normal. If the Euclidean vector norms $\|\boldsymbol{x}\|_{2}$ are used for each subspace $\Omega_{j}, 1 \leqq j \leqq N$, then each Gerschgorin set $G_{j}$ is the union of $n_{j}$ circles.

Proof. Let the eigenvalues of $A_{j, j}$ be $\sigma_{l}, 1 \leqq l \leqq n_{j}$. Since $A_{j, j}$ is normal, we can write $\left(||\left(A_{j, j}-z I_{j}\right)^{-1}||\right)^{-1}=\min _{l}\left|\sigma_{l}-z\right|$, which, combined with Definition 3, completes the proof.

It is quite simple to obtain the block analogues of well known results on inclusion regions for eigenvalues of $n \times n$ complex matrices. As a first example, the result of $A$. Brauer [2] on ovals of Cassini easily carries over.

Theorem 6. Let the $n \times n$ complex matrix $A$ be partitioned as in (2.1). Then, all the eigenvalues of $A$ lie in the union of the $[N(N-1)] / 2$ point sets $C_{i, j}$ defined by

$$
\left(\left\|\left(A_{i, i}-z I_{i}\right)^{-1}\right\| \cdot\left\|\left(A_{j, j}-z I_{j}\right)^{-1}\right\|\right)^{-1} \leqq\left(\sum_{\substack{l=1 \\ l \neq i}}^{N}\left\|A_{i, l}\right\|\right)\left(\sum_{\substack{l=1 \\ l \neq j}}^{N}\left\|A_{j, l}\right\|\right)
$$

where $1 \leqq i, j \leqq N$ and $i \neq j$. Moreover, if $A$ is block irreducible, and $\lambda$ is an eigenvalue of $A$ not in the interior of $\bigcup_{i \neq j} C_{i, j}$, then $\lambda$ is a boundary point of each of the point sets $C_{i, j}$.

Other obvious remarks can be made. Clearly, replacing $A$ by $A^{T}$ leaves the eigenvalues of $A$ invariant. Thus, rows sums can be replaced by column sums in the definition (2.4) of diagonal dominance, and many results using both row and column sums admit easy generalizations. As an illustration, we include the following known [4] generalization of a result by Ostrowski [9].

THEOREM. 7. Let the $n \times n$ complex matrix $A$ be partitioned as in, (2.1), and define 


$$
R_{j} \equiv \sum_{\substack{k=1 \\ k \neq j}}^{N}\left\|A_{j, k}\right\| ; \quad C_{j} \equiv \sum_{\substack{k=1 \\ k \neq j}}^{N}\left\|A_{k, j}\right\|, \quad 1 \leqq j \leqq N .
$$

Then, for any $\alpha$ with $0 \leqq \alpha \leqq 1$, each eigenvalue $\lambda$ of $A$ satisfies

$$
\left(\left\|\left(A_{j, j}-\lambda I_{j}\right)^{-1}\right\|\right)^{-1} \leqq R_{j}^{\alpha} C_{j}^{1-\alpha}
$$

for at least one $j, 1 \leqq j \leqq N$.

Also, the important result of Fan and Hoffman [3] carries over with ease.

THEOREM 8. Let the $n \times n$ complex matrix $A$ be partitioned as in (2.1). Let $p>1$, and $1 / p+1 / q=1$. If $\alpha>0$ satisfies

$$
\sum_{i=1}^{N}\left\{\frac{\left(\sum_{j \neq i}\left\|A_{i, j}\right\|\right)^{q}}{\left(\sum_{j \neq i}\left\|A_{i, j}\right\|^{p}\right)^{q / p}}\right\} \leqq \alpha^{q}\left(1+\alpha^{q}\right),
$$

(whenever $0 / 0$ occurs on the left-hand side, we agree to put $0 / 0=0$ ), then every eigenvalue $\lambda$ of $A$ satisfies at least one of the following relations:

$$
\left(\left\|\left(A_{j, j}-\lambda I_{j}\right)^{-1}\right\|\right)^{-1} \leqq \alpha\left(\sum_{\substack{k=1 \\ k \neq j}}^{N}\left\|A_{j, k}\right\|^{p}\right)^{1 / p}, \quad 1 \leqq j \leqq N .
$$

We wish to emphasize that, unlike the cases previously treated where all the matrices $A_{i, j}$ of (2.1) are $1 \times 1$ matrices, these new inclusion regions now depend on the vector norms used. It seems reasonable, at least theoretically, to minimize these inclusion regions by considering all possible vector norms to produce optimum results. Similarly, there is a great deal of flexibility in the manner in which the matrix $A$ is partitioned, and this perhaps can be used to advantage.

4. Another generalization. Another result, due again to Taussky [12], states that if an $n \times n$ matrix $A=\left(a_{i, j}\right)$ is strictly diagonally dominant in the usual sense of $\left(2.4^{\prime}\right)$ with positive real diagonal entries $a_{i, i} 1 \leqq i \leqq n$, then the eigenvalues $\lambda_{j}$ of $A$ satisfy

$$
R e \lambda_{j}>0, \quad 1 \leqq j \leqq n .
$$

Based on our previous results, we now give a generalization of this result which depends upon the use of absolute norms [1]. By this, we mean the following. First, if $\boldsymbol{x}$ is a column vector with complex components $x_{i}$, let $|\boldsymbol{x}|$ denote the vector with components $\left|x_{i}\right|$. If

$$
\|\boldsymbol{x}\|=\||\boldsymbol{x}|\|
$$

for all vectors $\boldsymbol{x}$, then the norm is an absolute norm. ${ }^{4}$ This is equivalent

\footnotetext{
${ }^{4}$ Clearly, the $l_{p}$-norms of footnote 2 are absolute norms.
} 
[1] to the property that if $|\boldsymbol{y}| \geqq|\boldsymbol{x}|$, i.e., each component of $|\boldsymbol{y}|-|\boldsymbol{x}|$ is a nonnegative real number, then

$$
\|\boldsymbol{y}\| \geqq\|\boldsymbol{x}\| \text {. }
$$

Next, if $B=\left(b_{i, j}\right)$ is a real $m \times m$ matrix with $b_{i, j} \leqq 0$ for all $i \neq j$, and if $B$ is nonsingular with $B^{-1} \equiv\left(r_{i, j}\right)$ such that $r_{i, j} \geqq 0$ for all $1 \leqq i$, $j \leqq m$, then $B$ is said to be an M-matrix [8].

THEOREM 9. Let the $n \times n$ complex matrix $A$ be partitioned as in (2.1), and let $A$ be block strictly diagonally dominant (or block irreducible and block diagonally dominant with strict inequality in (2.4) for at least one j). Further, assume that each submatrix $A_{j, j}$ is an M-matrix, $1 \leqq j \leqq N$, and the vector norms for each subspace $\Omega_{j}$ are absolute norms. If $\lambda$ is any eigenvalue of $A$, then

$$
\operatorname{Re} \lambda>0 \text {. }
$$

Proof. For simplicity, we shall consider again only the case where $A$ is block strictly diagonally dominant. Let $z$ be any complex number with $R e z \leqq 0$. If $A_{j, j}^{-1} \equiv\left(r_{k, l}\right)$, and $\left(A_{j, j}-z I_{j}\right)^{-1} \equiv\left(s_{k, l}(z)\right)$, it follows [8] from the assumption that $A_{j, j}$ is an $M$-matrix that

$$
\left|s_{k, l}(z)\right| \leqq r_{k, l},
$$$$
1 \leqq k, l \leqq n_{j}
$$

Next, with (4.4) and the assumption of absolute norms, it follows from (4.2) and (4.2') that

$$
\frac{\left\|\left(A_{j, j}-z I_{j}\right)^{-1} \boldsymbol{x}\right\|}{\|\boldsymbol{x}\|} \leqq \frac{\left\|A_{j, j}^{-1} \mid \boldsymbol{x}\right\| \|}{\|\boldsymbol{x}\|},
$$

so that from (2.3),

$$
\left(\left\|\left(A_{j, j}-z I_{j}\right)^{-1}\right\|\right)^{-1} \geqq\left(\left\|A_{j, j}^{-1}\right\|\right)^{-1} .
$$

In other words, for any $z$ with $R e z \leqq 0$, then the matrix $A-z I$ continues to be block strictly diagonally dominant, and hence nonsingular. Thus, if $\lambda$ is an eigenvalue of $A$, then $R e \lambda>0$, which completes the proof.

\section{BIBLIOGRAPHY}

1. F. L. Bauer, J. Stoer, and C. Witzgall, Absolute and monotonic norms, Numerische Math., 3 (1961), 257-264.

2. A. Brauer, Limits for the characteristic roots of a matrix II, Duke Math. J., 14 (1947), $21-26$.

3. Ky Fan and A. J. Hoffman, Lower bounds for the rank and location of the eigenvalues of a matrix, Contributions to the Solution of Systems of Linear Equations and the Determination of Eigenvalues, edited by Olga Taussky, Nat. Bur. of Standards Appl. Math. Series 39 (1954), 117-130. 
4. Miroslav Fiedler, Some estimates of spectra of matrices, Symposium on the Numerical Treatment of Ordinary Differential Equations, Integral and Integro-Differential Equations, Rome, 1960, Birkhauser Verlag, (1961), 33-36.

5. Miroslav Fiedler and Vlastimil Pták, Some inequalities for the spectrum of a matrix Mat. Fyz. Časopis. Slovensk. Akad. Vied, 10 (1960), 148-166.

6. Frank Harary, On the consistency of precedence matrices, J. Assoc. Comput. Mach., 7 (1960), 255-259.

7. Alston S. Householder, On the convergence of matrix iterations, J. Assoc. Comput. Mach, 3 (1956), 314-324.

8. A. M. Ostrowski, Über die Determinanten mit überwiegender Hauptdiagonale, Comment. Math. Helv., 10 (1937), 69-96.

9. A. M. Ostrowski, Über das Nichtverschwinden einer Klasse von Determinanten und die Lokalisierung der charakteristischen Wurzeln von Matrizen, Compositio Math., 9 (1951), 209-226.

10. A. M. Ostrowski, On some metrical properties of operator matrices and matrices partitioned into blocks, Journal of Math. Anal. and Appl., 2 (1961), 161-209.

11. Olga Taussky, Bounds for characteristic roots of matrices, Duke Math. J., 15 (1948). 1043-1044.

12. - A recurring theorem on determinants, Amer. Math. Monthly, 56 (1949), 672-676.

13. - Some topics concerning bounds for eigenvalues of finite matrices, Survey of Numerical Analysis, edited by John Todd, McGraw-Hill, (1962), 279-297. 


\section{PACIFIC JOURNAL OF MATHEMATICS}

\section{EDITORS}

\author{
Ralph S. Phillips \\ Stanford University \\ Stanford, California \\ M. G. Arsove \\ University of Washington \\ Seattle 5 , Washington
}

\author{
A. L. Whiteman \\ University of Southern California \\ Los Angeles 7, California \\ Lowell J. Paige \\ University of California \\ Los Angeles 24, California
}
E. F. BECKENBACH
D. DERRY
ASSOCIATE EDITORS
T. M. CHERRY
M. OHTSUKA
H. L. ROYDEN
E. G. STRAUS
E. SPANIER
F. WOLF

\section{SUPPORTING INSTITUTIONS}

\author{
UNIVERSITY OF BRITISH COLUMBIA \\ CALIFORNIA INSTITUTE OF TECHNOLOGY \\ UNIVERSITY OF CALIFORNIA \\ MONTANA STATE UNIVERSITY \\ UNIVERSITY OF NEVADA \\ NEW MEXICO STATE UNIVERSITY \\ OREGON STATE UNIVERSITY \\ UNIVERSITY OF OREGON \\ OSAKA UNIVERSITY \\ UNIVERSITY OF SOUTHERN CALIFORNIA
}

\author{
STANFORD UNIVERSITY \\ UNIVERSITY OF TOKYO \\ UNIVERSITY OF UTAH \\ WASHINGTON STATE UNIVERSITY \\ UNIVERSITY OF WASHINGTON \\ AMERICAN MATHEMATICAL SOCIETY \\ CALIFORNIA RESEARCH CORPORATION \\ SPACE TECHNOLOGY LABORATORIES \\ NAVAL ORDNANCE TEST STATION
}

Mathematical papers intended for publication in the Pacific Journal of Mathematıcs should be typewritten (double spaced), and the author should keep a complete copy. Manuscripts may be sent to any one of the four editors. All other communications to the editors should be addressed to the managing editor, L. J. Paige at the University of California, Los Angeles 24, California.

50 reprints per author of each article are furnished free of charge; additional copies may be obtained at cost in multiples of 50 .

The Pacific Journal of Mathematics is published quarterly, in March, June, September, and December. Effective with Volume 13 the price per volume (4 numbers) is $\$ 18.00$; single issues, $\$ 5.00$. Special price for current issues to individual faculty members of supporting institutions and to individual members of the American Mathematical Society: $\$ 8.00$ per volume; single issues $\$ 2.50$. Back numbers are available.

Subscriptions, orders for back numbers, and changes of address should be sent to Pacific Journal of Mathematics, 103 Highland Boulevard, Berkeley 8, California.

Printed at Kokusai Bunken Insatsusha (International Academic Printing Co., Ltd.), No. 6, 2-chome, Fujimi-cho, Chiyoda-ku, Tokyo, Japan.

PUBLISHED BY PACIFIC JOURNAL OF MATHEMATICS, A NON-PROFIT CORPORATION

The Supporting Institutions listed above contribute to the cost of publication of this Journal, but they are not owners or publishers and have no responsibility for its content or policies. 


\section{Pacific Journal of Mathematics}

\section{Vol. 12 , No. 4 \\ April, 1962}

Tsuyoshi Andô, On fundamental properties of a Banach space with a cone ..... . 1163

Sterling K. Berberian, A note on hyponormal operators ................ 1171

Errett Albert Bishop, Analytic functions with values in a Frechet space . . . . . . . 1177

(Sherman) Elwood Bohn, Equicontinuity of solutions of a quasi-linear equation ............................................ 1193

Andrew Michael Bruckner and E. Ostrow, Some function classes related to the class of convex functions . . . . . . . . . . . . . . . . . . . . . . . . 1203

J. H. Curtiss, Limits and bounds for divided differences on a Jordan curve in the complex domain . . ................................. 1217

P. H. Doyle, III and John Gilbert Hocking, Dimensional invertibility . . . . . . . . 1235

David G. Feingold and Richard Steven Varga, Block diagonally dominant matrices and generalizations of the Gerschgorin circle theorem ................. 1241

Leonard Dubois Fountain and Lloyd Kenneth Jackson, A generalized solution of the boundary value problem for $y^{\prime \prime}=f\left(x, y, y^{\prime}\right) \ldots \ldots \ldots \ldots \ldots \ldots \ldots \ldots \ldots$

Robert William Gilmer, Jr., Rings in which semi-primary ideals are primary. . . . . 1273

Ruth Goodman, K-polar polynomials .......................... 1277

Israel Halperin and Maria Wonenburger, On the additivity of lattice completeness ........................................... 1289

Robert Winship Heath, Arc-wise connectedness in semi-metric spaces . . . . . . . . 1301

Isidore Heller and Alan Jerome Hoffman, On unimodular matrices . . . . . . . . . . . 1321

Robert G. Heyneman, Duality in general ergodic theory . . . . . . . . . . . . . . . 1329

Charles Ray Hobby, Abelian subgroups of p-groups . . . . . . . . . . . . . . . 1343

Kenneth Myron Hoffman and Hugo Rossi, The minimum boundary for an analytic

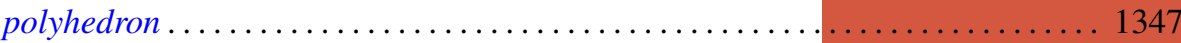

Adam Koranyi, The Bergman kernel function for tubes over convex cones ........ 1355

Pesi Rustom Masani and Jack Max Robertson, The time-domain analysis of a continuous parameter weakly stationary stochastic proces.

William Schumacher Massey, Non-existence of almost-complex structures on

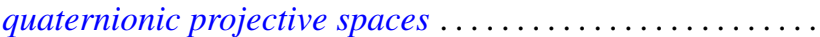

Deane Montgomery and Chung-Tao Yang, A theorem on the action of $\mathrm{SO}(3) \ldots . .1385$

Ronald John Nunke, A note on Abelian group extensions . . . . . . . . . . . . . 1401

Carl Mark Pearcy, A complete set of unitary invariants for operators generating finite $W^{*}$-algebras of type $I$

Edward C. Posner, Integral closure of rings of solutions of linear differential equations.

Duane Sather, Asymptotics. III. Stationary phase for two parameters with an application to Bessel functions.

J. Śladkowska, Bounds of analytic functions of two complex variables in domains

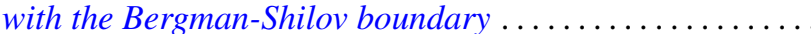

Joseph Gail Stampfli, Hyponormal operators .

George Gustave Weill, Some extremal properties of linear combinations of kernels

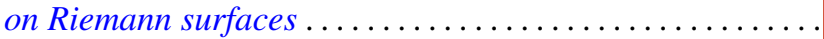

